\title{
Retrospective analysis of the clinicopathological characteristics of gastrointestinal neuroendocrine neoplasms
}

\author{
ZHIQIANG WANG ${ }^{1}$, WENLIANG LI ${ }^{1}$, TIANXING CHEN ${ }^{2}$, JUN YANG ${ }^{1}$, \\ LILIN LUO ${ }^{2}$, LIANYU ZHANG ${ }^{3}$, BAOCUN SUN ${ }^{3}$ and RUI LIANG ${ }^{2}$ \\ ${ }^{1}$ Department of Oncology, The First Affiliated Hospital of Kunming Medical University; ${ }^{2}$ Department of Pathology, \\ The First People's Hospital of Yunnan Province, Kunming, Yunnan 650032; ${ }^{3}$ Department of Pathology, \\ The Affiliated Cancer Hospital of Tianjin Medical University, Tianjin 300060, P.R. China
}

Received November 1, 2014; Accepted June 22, 2015

DOI: $10.3892 /$ etm.2015.2634

\begin{abstract}
The aim of the present study was to analyze and summarize the clinicopathological characteristics and factors affecting prognosis for patients with gastrointestinal neuroendocrine neoplasms (GINENs). Retrospective analysis was conducted on the clinicopathological data of 74 patients who were diagnosed with GINEN, and immunohistochemical methods were used to detect the expression levels of relevant markers [synaptophysin (Syn), chromogranin A (CgA) and Ki-67]. Among the 74 cases with GINEN, there were 39 males and 35 females, with an average age of 56.9 years. There were 32 neoplasms in the rectum, 29 in the stomach, 6 in the colon, 2 in the small intestine and 5 in the appendix. All 74 cases underwent surgical resection. According to the World Health Organization Classification of Tumors of the Digestive System (2010), the diagnosis of the 74 cases showed 41 cases $(55.4 \%)$ of neuroendocrine tumor (NET; 25 cases of G1 and 16 cases of G2), 21 cases $(28.4 \%)$ of neuroendocrine carcinoma (NEC) and 12 cases $(16.2 \%)$ of mixed adenoneuroendocrine carcinoma (MANEC). Additionally, 19 cases had metastasis to lymph nodes. During 10-34 months of follow-up, 15 patients had distant metastasis and 24 patients succumbed, and the accumulative survival rate in 1 or 2 years was 87.8 and $74.3 \%$, respectively. Six factors, namely neoplasm size, depth of invasion, lymph node metastasis, distant metastasis, pathological type and the expression or lack of expression of $\mathrm{CgA}$, significantly affected the survival time of patients. Definitive diagnosis of GINEN mainly relies on pathological diagnosis. GINENs with different histopathological types and grading have different clinicopathological characteristics and prognosis: NETs are mainly early lesions with a good prognosis,
\end{abstract}

Correspondence to: Dr Rui Liang, Department of Pathology, The First People's Hospital of Yunnan Province, 157 Jinbi Road, Kunming, Yunnan 650032, P.R. China

E-mail: ruiliang131@hotmail.com

Key words: neuroendocrine neoplasm, gastrointestinal tract, pathology, retrospective analysis whereas NECs and MANECs have high malignancy and strong invasion with a worse prognosis.

\section{Introduction}

Gastrointestinal neuroendocrine neoplasms (GINENs), originating from amine precursor uptake and decarboxylation cells in the digestive tract, have the ability to undergo multiple differentiation and secrete various active hormones, leading to significant differences in biological behaviors and prognosis (1). Previous studies have indicated that the incidence rate of GINEN is low (2); however, an epidemiological investigation in the USA showed that the incidence rate was 5.25/100,000 in 2004, with a 5-fold increase from that 30 years earlier (3). The original denomination and classification for GINEN was disorganized and non-uniform, and knowledge concerning the neoplasms in clinical practice was very limited. Therefore, the World Health Organization (WHO) Classification of Tumors of the Digestive System (4) revised the denomination and classification for GINEN, dividing it into neuroendocrine tumor (NET), neuroendocrine carcinoma (NEC), mixed adenoneuroendocrine carcinoma (MANEC), hyperplasia and pre-neoplasm (4). There are limited statistical data regarding the incidence and pathology of GINENs in Chinese patients.

In the present study, the clinicopathological data from 74 patients with GINEN were retrospectively analyzed, with the aim of summarizing and analyzing the clinicopathological characteristics of GINEN and the factors affecting prognosis, and thereby to help in improving the understanding of these neoplasms.

\section{Subjects and methods}

Patients. Intact data of 74 cases with GINEN (from January 2012 to December 2013) confirmed by pathological examination from the Affiliated Cancer Hospital of Tianjin Medical University (Tianjin, China) were retrospectively reviewed. Among them, 39 cases were male and 35 were female, with a gender ratio of 1.15:1. The ages of the patients ranged from 30 to 73 years, and the average age was 56.9 years. In total, 32 of the neoplasms were located in the rectum $(43.2 \%)$, 29 in the stomach (39.2\%), 6 in the colon (8.1\%), 2 in the small 
Table I. Expression of neuroendocrine markers and Ki-67 in different types of gastrointestinal neuroendocrine neoplasms.

\begin{tabular}{|c|c|c|c|c|c|c|c|c|}
\hline \multirow[b]{2}{*}{ Group } & \multirow[b]{2}{*}{ Cases } & \multicolumn{4}{|c|}{ Neuroendocrine markers, n (\%) } & \multicolumn{3}{|c|}{ Ki-67 expression levels, n (\%) } \\
\hline & & $\mathrm{Syn}^{+}$ & $\mathrm{CgA}^{+}$ & $\mathrm{Syn}^{+}$or $\mathrm{CgA}^{+}$ & $\mathrm{Syn}^{+} \mathrm{CgA}^{+}$ & $\leq 2 \%$ & $3-20 \%$ & $>20 \%$ \\
\hline NET G1 & 25 & $22(88.0)$ & $18(72.0)$ & $25(100)$ & $16(64.0)$ & $25(100.0)$ & 0 & 0 \\
\hline NET G2 & 16 & $14(87.5)$ & $10(62.5)$ & $16(100)$ & $9(56.3)$ & $1(6.2)$ & $15(93.8)$ & 0 \\
\hline NEC & 21 & $21(100)$ & $10(47.6)$ & $21(100)$ & $10(47.6)$ & 0 & 0 & $21(100)$ \\
\hline MANEC & 12 & $12(100)$ & $6(50.0)$ & $12(100)$ & $6(50.0)$ & 0 & 0 & $12(100)$ \\
\hline Total & 74 & 69 (93.2) & $44(59.5)$ & $74(100)$ & $41(55.4)$ & $26(35.1)$ & $15(20.3)$ & $33(44.6)$ \\
\hline
\end{tabular}

Syn, synaptophysin; CgA, chromogranin A; NET, neuroendocrine tumor; NEC, neuroendocrine carcinoma; MANEC, mixed adenoneuroendocrine carcinoma.

intestine $(2.7 \%)$ and 5 in the appendix $(6.8 \%)$. This study was conducted in accordance with the Declaration of Helsinki, and with the approval of the Ethics Committee of the Affiliated Cancer Hospital of Tianjin Medical University. Written informed consent was obtained from all the participants.

Symptoms. All but two of the patients manifested non-functional symptoms of GINEN. These were mainly non-specific digestive tract symptoms, for example, progressive dysphagia, abdominal pain, abdominal distention, diarrhea, constipation, abdominal mass, bloody stools or melena, anorexia and weight loss, and 2 patients had carcinoid syndrome, for example, facial flushing, diarrhea and excessive perspiration. The remaining 2 patients had gastrinoma-manifested functional symptoms, for example, intractable peptic ulcer, upper abdominal pain and diarrhea.

Histopathological examination. Following surgery, the tissues were sent for routine histopathological examination. Two pathologists reviewed all the pathological sections according to the WHO Classification of Tumors of the Digestive System (4).

Immunopathological examination. Mouse anti-human pan-cytokeratin (PCK, AE1/AE3; MAB-0049), low molecular weight cytokeratin (LCK, 35 $\beta$ H11; MAB-0051) and Ki-67 (MIB-1; MAB-0129) monoclonal antibodies, and rabbit anti-human synaptophysin (Syn, SP11; RMA-0537) and chromogranin A (CgA, SP12; RMA-0548) for immunohistochemistry were purchased from Maixin Biotechnology Inc. (Fuzhou, China). Polymeric horseradish peroxidase conjugated anti-mouse/rabbit IgG (Maixin Biotechnology Inc.) was used as the secondary antibody. The diagnostic basis for GINEN was that at least one of the neuroendocrine markers was diffusely positive or strongly positive; the partial expression of several neuroendocrine markers was not considered to diagnose GINEN.

Surgery. All 74 cases underwent surgery: 30 cases $(40.5 \%)$ where the maximum diameters of the neoplasms were $<2 \mathrm{~cm}$ underwent endoscopic mucosal dissection; 9 cases $(12.2 \%)$ underwent partial gastrectomy or partial enterectomy; and 35 cases $(47.3 \%)$ underwent radical excision.
Postoperative therapy. Of the 74 patients, 5 patients received postoperative chemotherapy with cisplatin and etoposide, 4 patients with oxaliplatin and 5-fluorouracil, and 3 patients with oxaliplatin and capecitabine. Two patients received postoperative biotherapy with octreotide, and 3 patients received postoperative biotherapy and targeted therapy with octreotide and bevacizumab.

Statistical analysis. SPSS software (version 13.0; SPSS Inc., Chicago, IL, USA) was applied for statistical analysis. Comparisons of two or multiple sample survival rates were performed using $\chi^{2}$ test, and the differences of survival rate among different groups were performed using log-rank. $\mathrm{P}<0.05$ was considered to indicate a statistically significant difference.

\section{Results}

Histopathological examination. Two pathologists reviewed all the pathological sections and provided a unified diagnosis. All 74 cases were diagnosed with GINEN. Typing and grading: i) NET, 41 cases $(55.4 \%)$, among which 25 cases $(33.8 \%)$ were at G1 and 16 cases (21.6\%) were at G2; ii) NEC, 21 cases, among which 8 cases were small cell carcinoma and 13 cases were large cell carcinoma; iii) MANEC, 12 cases (16.2\%), among which 5 cases were low-differentiation adenocarcinoma with NEC, 1 case was middle-differentiation adenocarcinoma with NET G2, 4 cases were mucinous adenocarcinoma with NEC and 2 cases were signet-ring cell carcinoma with NEC as shown in Fig. 1.

Clinicopathological characteristics. The maximum diameters of the tumors varied between 0.5 and $12 \mathrm{~cm}$, with an average of $4.1 \mathrm{~cm}$. The depth of the tumor invasion was the mucous or sub-mucous layer in 33 cases $(44.6 \%)$ and the muscular layer and serosa in 41 cases $(55.4 \%)$. Lymph node metastasis occurred in 19 cases $(25.7 \%)$.

Immunopathological examination. All 74 cases of GINEN underwent routine immunohistochemical examination. The proportions of cases with positive expression of PCK and LCK were 83.8 and $87.8 \%$, respectively. All of the GINENs (100\%) were positive for least one of PCK and LCK. The expression 
A

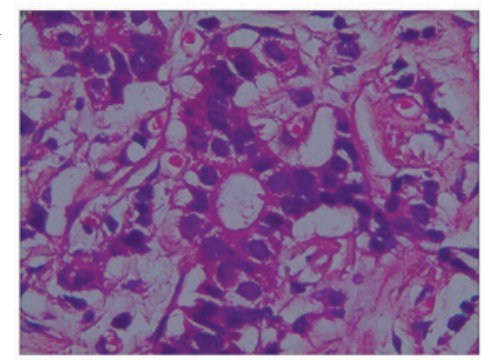

D

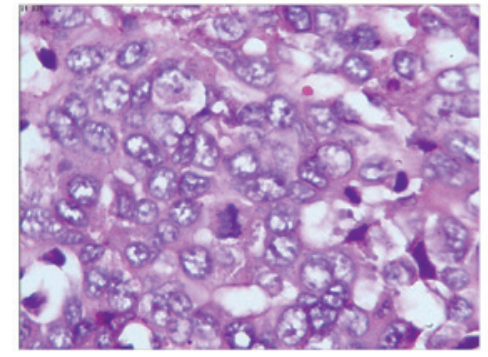

B

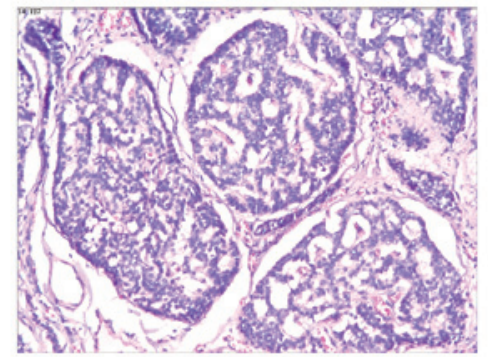

E

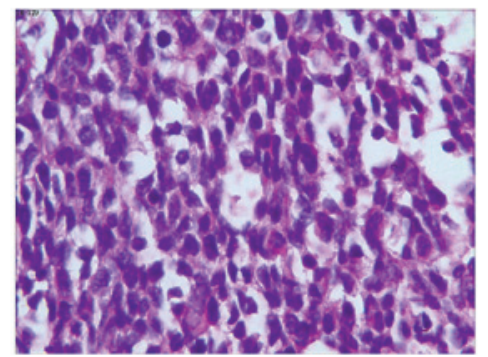

$\mathrm{C}$

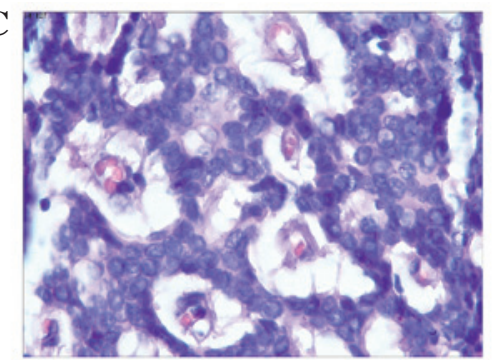

F

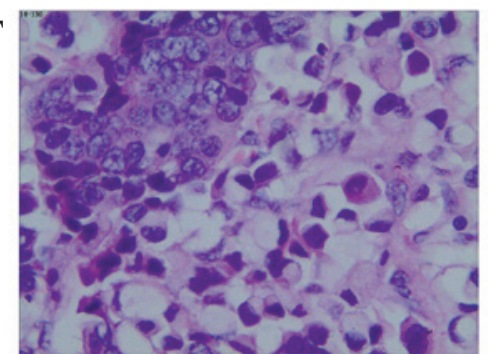

Figure 1. Different types of gastrointestinal neuroendocrine neoplasms with hematoxylin and eosin staining. (A) Appendix, NET G1 (magnification, x200); (B) rectal, NET G2 (magnification, x40): (C) rectum, NET G2 (magnification, x200); (D) stomach, NEC, large cell carcinoma, (magnification, x200); (E) colon, NEC, small cell carcinoma (magnification, x200); (F) stomach, mixed adenoneuroendocrine carcinoma (magnification, x200), NET, neuroendocrine tumor; NEC, neuroendocrine carcinoma.

A

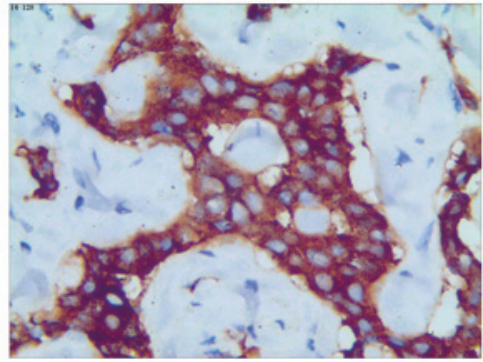

C

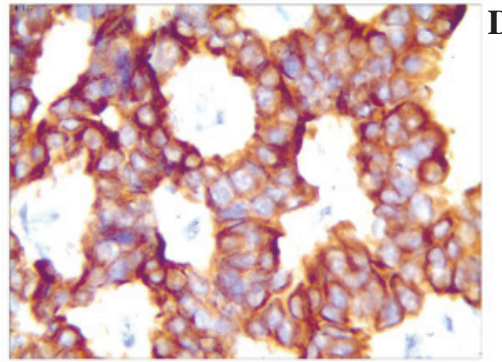

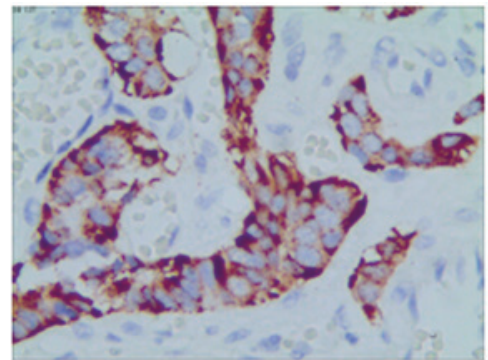

D

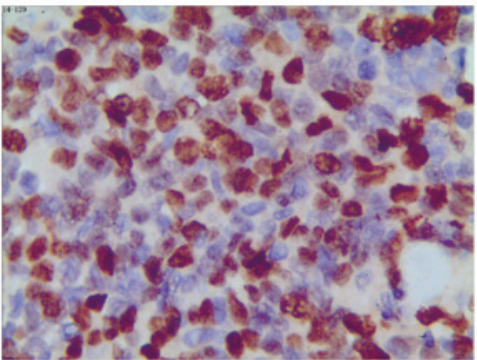

Figure 2. Immunohistochemical staining in different types of gastrointestinal neuroendocrine neoplasms. (A) Appendix, NET G1, Syn; (B) small intestine, NET G2, CgA; (C) Rectum, NET G2, PCK; (D) Rectum, NEC, small cell carcinoma, Ki-67 (all magnification, x200). NET, neuroendocrine tumor; NEC, neuroendocrine carcinoma; Syn, synaptophysin; CgA, chromogranin A; PCK, pan-cytokeratin.

rates of Syn and $\mathrm{CgA}$ were 93.2 and $59.5 \%$, respectively, and Ki-67 showed different expression levels depending on the tumor type (Table I and Fig. 2).

Follow-up examination. Among the 74 patients with GINEN, 71 cases were followed-up for 10-34 months. During follow-up, 15 cases had distant metastasis and 24 patients succumbed. The survival rate after 1 and 2 years was 87.8 and $74.3 \%$, respectively. The results showed that gender, age or neoplasm site did not have a significant effect on survival time; however, the survival rate after 1 and 2 years for patients with a neoplasm diameter of $\leq 2 \mathrm{~cm}$ was significantly higher than that of patients with larger neoplasms
$(\mathrm{P}=0.002$ and $\mathrm{P}<0.001$, respectively). The survival rate after 1 and 2 years for patients with neoplasms involving mucous or sub-mucous layers was significantly higher than that for neoplasms involving the muscular layer or serosa $(\mathrm{P}=0.004$ and $\mathrm{P}<0.001$, respectively). The survival rate after 1 and 2 years for patients with lymph node metastasis was significantly lower than without ( $\mathrm{P}<0.001$ for both). The survival rate after 1 and 2 years for patients with distant metastasis was significantly lower than patients without distant metastasis $(\mathrm{P}=0.002$ and $\mathrm{P}<0.001$, respectively), and the survival rate after 1 and 2 years for patients with $\mathrm{CgA}$ expression was significantly higher than that for patients without $\mathrm{CgA}$ expression ( $\mathrm{P}=0.026$ and $\mathrm{P}=0.006$, respectively). The differ- 
Table II. Factors affecting the survival rates of patients with gastrointestinal neuroendocrine neoplasms.

\begin{tabular}{lcc}
\hline & \multicolumn{2}{c}{ Survival rate (\%) } \\
\cline { 2 - 3 } Factors & 1 year & 2 year \\
\hline Neoplasm size, cm & & \\
$\leq 2$ & $33 / 33(100)$ & $33 / 33(100)$ \\
$2.1-5$ & $17 / 19(89.5)$ & $13 / 19(68.4)$ \\
$>5$ & $15 / 22(68.2)$ & $9 / 22(40.9)$ \\
Depth of invasion & & \\
Mucous or sub-mucous layer & $33 / 33(100)$ & $32 / 33(97.0)$ \\
Muscular layer or serosa & $32 / 41(78.0)$ & $23 / 41(56.1)$ \\
Lymph node metastasis & & \\
Without & $53 / 55(96.4)$ & $49 / 55(89.1)$ \\
With & $12 / 19(63.2)$ & $6 / 19(31.6)$ \\
Distant metastasis & & \\
Without & $56 / 59(94.9)$ & $53 / 59(89.8)$ \\
With & $9 / 15(60.0)$ & $2 / 15(13.3)$ \\
Histopathological types & & \\
NET G1 & $25 / 25(100)$ & $25 / 25(100)$ \\
NET G2 & $16 / 16(100)$ & $14 / 16(87.5)$ \\
NEC & $15 / 21(71.4)$ & $10 / 21(47.6)$ \\
MANEC & $9 / 12(75.0)$ & $6 / 12(50.0)$ \\
CgA expression & & \\
Positive & & \\
Negative & $42 / 44(95.5)$ & $38 / 44(86.4)$ \\
\hline
\end{tabular}

NET, neuroendocrine tumor; NEC, neuroendocrine carcinoma; MANEC, mixed adenoneuroendocrine carcinoma; CgA, chromogranin A.

ence of survival rate after 1 and 2 years between patients with NET G1 and NET G2 was not statistically significant ( $\mathrm{P}=0.07$ and $\mathrm{P}=0.146$, respectively); nor was that for $\mathrm{NEC}$ and MANEC $(\mathrm{P}=0.825$ and $\mathrm{P}=0.895$, respectively). However, the survival rate after 1 and 2 years for patients with NET (including G1 and G2) was significantly higher than that for patients with NEC and MANEC (both $\mathrm{P}<0.001$; Table II).

\section{Discussion}

The reported incidence rate of GINEN is significantly lower than that of gastrointestinal adenocarcinoma, accounting for $0.4-1.8 \%$ of gastrointestinal malignant tumors (5). With the popularization of gastrointestinal endoscopy and the development of immunopathology, the overall incidence rate for GINEN has been rising in the last few years (6-8). GINEN is the most common type of neuroendocrine neoplasm (NEN), and $67.5 \%$ of NEN originates from the gastrointestinal tract (9). Previously, it was considered that GINEN mainly affected the appendix. but Maggard et al (10) analyzed 11,427 cases of GINEN and showed that the small intestine was the most common site $(44.7 \%)$, followed by the rectum $(19.6 \%)$, appendix $(16.7 \%)$, colon (10.6\%) and stomach (7.2\%). In 2008, data from the USA showed that the most common sites of GINEN were the rectum, jejunum and stomach (3). In 2010 (11), data from the National Registration Center of Spain showed that the jejunum-ileum was the most common site of GINEN. In the present study, the most common sites were the rectum and stomach, which is significantly different from other reports $(3,10,11)$, possibly as a result of differences in nationalities or sample sizes.

Since the differentiation of GINEN from gastrointestinal adenocarcinoma on the basis of clinical symptoms and endoscopic and ultrasonic morphologies is challenging, the diagnosis of GINEN principally depends on pathological examination. However, GINEN has complex and various histomorphological manifestations, and its pathological diagnosis criteria, denomination and classification have experienced some revision. In 1907, Oberndorfer (12) proposed the term 'carcinoid' for GINEN, which was regarded as a benign tumor similar to carcinoma. Subsequent studies, however, showed that GINEN may be malignant and metastasize. In 1963, based on its embryological origin, 'carcinoid' was simply divided into neoplasms of the anterior intestines (lung, stomach, duodenum, proximal jejunum and pancreas), middle intestines (distal jejunum, ileum, appendix and caecum) and posterior intestines (colon and rectum) (13). In 1980, the WHO classification of Tumors of the Digestive System (2nd revision) designated all NENs as 'carcinoid.' In 2000, the WHO classification (3rd revision) divided digestive system NEN into 5 primary types: Well-differentiated endocrine tumor, well-differentiated neuroendocrine carcinoma, poorly differentiated endocrine carcinoma, small cell carcinoma and tumor-like lesion. In 2010, the WHO Classification was further improved to divide digestive system NEN into 6 types: NET G1, NET G2, NEC, MANEC, hyperplasia and pre-neoplasia (4). Typing and grading-scale systems depend on pathological histology, pathological mitosis and the Ki-67 index. When pathological mitosis grading is not consistent with Ki-67 index grading, the highest grading between the two is taken as their grading. In the present study, one case had a Ki-67 index of $\leq 2 \%$ but a pathological mitosis rate of $5 / 10$ high-power fields; therefore, the pathological diagnosis was NET G2.

With regard to immunohistochemistry, the present study considered that at least one neuroendocrine marker being diffusely positive or strongly positive was diagnostic for GINEN. In this study, the proportions of cases with positive expression of Syn, CgA and Syn $+\mathrm{CgA}$ were 93.2, 59.5 and $55.4 \%$, respectively. In addition, 28 cases were $\mathrm{Syn}^{+} \mathrm{CgA}^{-}$, and 3 cases were $\mathrm{CgA}^{+} \mathrm{Syn}^{-}$, which suggested that the expression spectrum of Syn and CgA did not entirely overlap with each other, and any single indicator was not perfect; hence, two or more neuroendocrine markers should be combined to improve the accuracy of GINEN diagnosis. The above-mentioned results were in line with the report of Hirabayashi et al (14).

Furthermore, several factors can affect the prognosis of patients with GINEN. During follow-up (10-34 months), the survival rate after 1 or 2 years was 87.8 and $74.3 \%$, respectively. Through analysis of follow-up data, it was found that gender, age or neoplasm site did not significantly influence the survival time of the patients. Certain studies, however, have shown the survival rate after 5 years of patients with GINEN in the rectum or appendix to be significantly higher than that of patients with GINEN in the stomach or colon $(3,15,16)$. This differs from what the present study showed, which may be due 
to a small sample size and shorter duration of follow-up in the present study. Patients with a small neoplasm size, shallow invasion, no lymph node metastasis, a low pathological grading and no expression of $\mathrm{CgA}$ have a better prognosis, which is in line with previous reports (17-21).

Currently, surgery is the preferred treatment for patients with GINEN (22-24). Regardless of whether or not metastasis has occurred, surgery dependent upon neoplasm size, site and depth of invasion is required to resect the primary site of the neoplasm, any sites of metastasis and lymph nodes in order to improve survival rates (23-26). Other treatments for GINEN include chemotherapy, biotherapy and molecular targeted therapy $(27,28)$. Chemotherapy is mainly used to treat patients with NEC or MANEC, while NETs have low sensitivity to chemotherapy (29,30). Biotherapy and molecular targeted therapy have good prospects in the treatment of patients with progressive NET (31). In the present study, all 74 cases of GINEN underwent surgery with different surgical scope; 12 cases underwent postoperative chemotherapy; and 5 cases underwent postoperative biotherapy and molecular targeted therapy. Due to the relatively short duration of follow-up, it was challenging to adequately evaluate therapeutic schemes; however, GINEN, with a superior prognosis, has slower progression than gastrointestinal adenocarcinoma.

Future studies should expand the sample size and follow-up time period in order to more accurately summarize the pathological and immunohistochemical features of GINEN, promote pathologists' understanding of the neoplasm and improve the diagnostic accuracy of GINEN and its subtypes. Furthermore, the factors affecting the prognosis of patients with GINEN may be more fully explored and therapeutic schemes compared, in order to raise awareness of GINEN and bring the benefit of comprehensive treatment for GINEN to clinical practice.

\section{References}

1. Li CC, Xu B, Hirokawa M, Qian Z, Yoshimoto K, Horiguchi H, Tashiro T and Sano T: Alterations of E-cadherin, alpha-catenin and beta-catenin expression in neuroendocrine tumors of the gastrointestinal tract. Virchows Arch 440: 145-154, 2002.

2. Scherübl H, Streller B, Stabenow R, Herbst H, Höpfner M, Schwertner C, Steinberg J, Eick J, Ring W, Tiwari K and Zappe SM: Clinically detected gastroenteropancreatic neuroendocrine tumors are on the rise: Epidemiological changes in Germany. World J Gastroenterol 19: 9012-9019, 2013.

3. Yao JC, Hassan M, Phan A, Dagohoy C, Leary C, Mares JE, Abdalla EK, Fleming JB, Vauthey JN, Rashid A and Evans DB: One hundred years after 'carcinoid': Epidemiology of and prognostic factors for neuroendocrine tumors in 35,825 cases in the United States. J Clin Oncol 26: 3063-3072, 2008.

4. Bosman FT, Carneiro F, Hruban RH and Theise ND (eds): World Health Organization Classification of Tumours of the Digestive System. Vol. 3. 4th edition. IARC Press, Lyon, 2010.

5. Shebani KO, Souba WW, Finkelstein DM, Stark PC, Elgadi KM, Tanabe KK and Ott MJ: Prognosis and survival in patients with gastrointestinal tract carcinoid tumors. Ann Surg 229: 815-821, 1999.

6. Buitrago D, Trencheva K, Zarnegar R, Finnerty B, Aldailami H, Lee SW, Sonoda T, Milsom JW and Fahey TJ III: The impact of incidental identification on the stage at presentation of lower gastrointestinal carcinoids. J Am Coll Surg 213: 652-656, 2011.

7. Federspiel BH, Hansen CP, Vainer B, Hasselby JP, Bardram L and Knigge U: Incidence, pathology and clinical course and symptoms of neuroendocrine gastrointestinal tumours. Ugeskr Laeger 172: 2946-2950, 2010 (In Danish)

8. Lawrence B, Gustafsson BI, Chan A, Svejda B, Kidd M and Modlin IM: The epidemiology of gastroenteropancreatic neuroendocrine tumors. Endocrinol Metab Clin North Am 40: 1-18, 2011.
9. Salyers WJ, Vega KJ, Munoz JC, Trotman BW and Tanev SS: Neuroendocrine tumors of the gastrointestinal tract: Case reports and literature review. World J Gastrointest Oncol 6: 301-310, 2014

10. Maggard MA, O'Connell JB and Ko CY: Updated populationbased review of carcinoid tumors. Ann Surg 240: 117-122, 2004.

11. Garcia-Carbonero R, Capdevila J, Crespo-Herrero G, DíazPérez JA, Martínez Del Prado MP, Alonso Orduña V, Sevilla-García I, Villabona-Artero C, Beguiristain-Gómez A, Llanos-Muñoz M, et al: Incidence, patterns of care and prognostic factors for outcome of gastroenteropancreatic neuroendocrine tumors (GEP-NETs): Results from the National Cancer Registry of Spain (RGETNE). Ann Oncol 21: 1794-1803, 2010.

12. Oberndorfer S: Carcinoid tumors of the duodenum. Frankfurter Zeitschrift für Pathologie 1: 426-432, 1907 (In German)

13. Williams ED and Sandler M: The classification of carcinoid tumours. Lancet 1: 238-239, 1963.

14. Hirabayashi K, Zamboni G, Nishi T, Tanaka A, Kajiwara H and Nakamura N: Histopathology of gastrointestinal neuroendocrine neoplasms. Front Oncol 3: 2, 2013.

15. Weinstock B, Ward SC, Harpaz N, Warner RR, Itzkowitz S and Kim MK: Clinical and prognostic features of rectal neuroendocrine tumors. Neuroendocrinology 98: 180-187, 2013.

16. Landry CS, Brock G, Scoggins CR, McMasters KM and Martin RC II: Proposed staging system for colon carcinoid tumors based on an analysis of 2,459 patients. J Am Coll Surg 207: 874-881, 2008.

17. Medrano-Guzmán R,Alvarado-Cabrero I,González-RodríguezD, López-García SC and Páez-Agraz F: Surgical prognostic factors of gastroenteropancreatic neuroendocrine tumors (GEP NET). Rev Med Inst Mex Seguro Soc 50: 243-248, 2012 (In Spanish).

18. Lim T, Lee J, Kim JJ, Lee JK, Lee KT, Kim YH, Kim KW, Kim S, Sohn TS, Choi DW, et al: Gastroenteropancreatic neuroendocrine tumors: Incidence and treatment outcome in a single institution in Korea. Asia Pac J Clin Oncol 7: 293-299, 2011.

19. Curran T, Tulin-Silver S, Patel K, Ward S, Schneiderman M, Harpaz N, Schwartz M, Itzkowitz S, Warner RR and Kim MK: Prognostic clinicopathologic factors in longitudinally followed patients with metastatic small bowel carcinoid tumors. Pancreas 40: 1253-1257, 2011.

20. Chou WC, Hung YS, Hsu JT, Chen JS, Lu CH, Hwang TL, Rau KM, Yeh KY, Chen TC and Sun CF: Chromogranin A is a reliable biomarker for gastroenteropancreatic neuroendocrine tumors in an Asian population of patients. Neuroendocrinology 95: 344-350, 2012.

21. Chou WC, Chen JS, Hung YS, Hsu JT, Chen TC, Sun CF, Lu CH and Hwang TL: Plasma chromogranin A levels predict survival and tumor response in patients with advanced gastroenteropancreatic neuroendocrine tumors. Anticancer Res 34: 5661-5669, 2014.

22. Knigge U and Hansen CP: Surgery for GEP-NETs. Best Pract Res Clin Gastroenterol 26: 819-831, 2012.

23. Fendrich V and Bartsch DK: Surgical treatment of gastrointestinal neuroendocrine tumors. Langenbecks Arch Surg 396: 299-311, 2011.

24. Huang LC, Poultsides GA and Norton JA: Surgical management of neuroendocrine tumors of the gastrointestinal tract. Oncology (Williston Park) 25: 794-803, 2011.

25. Ohtsuka T, Takahata S, Ueda J, Ueki T, Nagai E, Mizumoto K, Shimizu S and Tanaka M: Surgical treatment of gastroentero-pancreatic neuroendocrine tumor. Gan To Kagaku Ryoho 40: 843-846, 2013 (In Japanese).

26. Gaujoux S, Sauvanet A and Belghiti J: Place of surgical resection in the treatment strategy of gastrointestinal neuroendocrine tumors. Target Oncol 7: 153-159, 2012.

27. Scherübl H, Jensen RT, Cadiot G, Stölzel U and Klöppel G: Management of early gastrointestinal neuroendocrine neoplasms. World J Gastrointest Endosc 3: 133-139, 2011.

28. Pavel ME, Hainsworth JD, Baudin E, Peeters M, Hörsch D, Winkler RE, Klimovsky J, Lebwohl D, Jehl V, Wolin EM, et al; RADIANT-2 Study Group: Everolimus plus octreotide long-acting repeatable for the treatment of advanced neuroendocrine tumours associated with carcinoid syndrome (RADIANT-2): A randomised, placebo-controlled, phase 3 study. Lancet 378: 2005-2012, 2011.

29. Reidy DL, Tang LH and Saltz LB: Treatment of advanced disease in patients with well-differentiated neuroendocrine tumors. Nat Clin Pract Oncol 6: 143-152, 2009.

30. Khasraw M, Yap SY and Ananda S: Neuroendocrine neoplasms of the GI tract: The role of cytotoxic chemotherapy. Expert Rev Anticancer Ther 13: 451-459, 2013.

31. Castellano D, Bajetta E, Panneerselvam A, Saletan S, Kocha W, O'Dorisio T, Anthony LB and Hobday T; RADIANT-2 Study Group: Everolimus plus octreotide long-acting repeatable in patients with colorectal neuroendocrine tumors: A subgroup analysis of the phase III RADIANT-2 study. Oncologist 18: 46-53, 2013. 